Zbigniew Grande, Institute of Mathematics, Pedagogical University, plac Weyssenhoffa 11, 85-072, Bydgoszcz, Poland

e-mail: grande@wsp. bydgoszcz.pl

\title{
ON CONTINUITY AND GENERALIZED CONTINUITY WITH RESPECT TO TWO TOPOLOGIES
}

\begin{abstract}
Let $\tau_{1}$ and $\tau_{2}$ be topologies in $X$ and let $\tau=\tau_{1} \cap \tau_{2}$. Some conditions concerning the topologies $\tau, \tau_{1}$ and $\tau_{2}$ and describing the relations between the $\tau$-continuity (quasicontinuity) [cliquishness] and the $\tau_{i}$-continuity (quasicontinuity) [cliquishness], $i=1,2$, of functions defined on $X$ are considered.
\end{abstract}

Let $\mathcal{R}$ denote the set of all reals and let $(X, \tau)$ be a topological space.

A function $f: X \mapsto \mathcal{R}$ is called $\tau$-quasicontinuous ( $\tau$-cliquish) at a point $x \in X$ ([3] if for every positive real $\eta$ and for every set $U \in \tau$ containing $x$ there is a nonempty set $V \in \tau$ such that $V \subset U$ and $|f(v)-f(x)|<\eta$ for all points $v \in V\left(\operatorname{osc}_{V} f<\eta\right.$, where $\operatorname{osc}_{V} f$ denotes the diameter of the set $\left.f(V)\right)$.

We will consider two topologies $\mathcal{T}_{1}$ and $\mathcal{T}_{2}$ in $X$. Let

$$
\mathcal{T}=\mathcal{T}_{1} \cap \mathcal{T}_{2}
$$

\section{$1 \mathcal{T}$ - and $\mathcal{T}_{i}$-continuity}

Obviously, if a function $f: X \mapsto \mathcal{R}$ is $\mathcal{T}$-continuous at a point $x \in X$ then it is also $\mathcal{T}_{i}$-continuous at $x$ for $i=1,2$. The converse implication need not be valid.

Example 1. Let

$$
U=\bigcup_{n}\left[\frac{1}{4 n+1}, \frac{1}{4 n}\right] \cup\{0\}
$$

Key Words: bitopological continuity, quasicontinuity, cliquishness

Mathematical Reviews subject classification: Primary: 54C08, 54C30, 54C05

Received by the editors September 17, 1997

* Supported by Bydgoszcz Pedagogical University grant 1997 
and

$$
V=\bigcup_{n}\left[\frac{1}{4 n+3}, \frac{1}{4 n+2}\right] \cup\{0\} .
$$

Denote by $\mathcal{T}_{1}$ the topology of subsets of $X=\mathcal{R}$ generated by the family

$$
\{(-r, r) \cap U ; r>0\}
$$

and by $\mathcal{T}_{2}$ the topology of subsets of $X$ generated by the family

$$
\{(-r, r) \cap V: r>0\}
$$

For $n=1,2, \ldots$, let

$$
I_{n}=\left[\frac{1}{4 n+2}, \frac{1}{4 n+1}\right]
$$

and let $f_{n}: I_{n} \mapsto[0,1]$, be a continuous function (with respect to the Euclidean topology $\mathcal{T}_{e}$ ) such that

$$
f_{n}\left(I_{n}\right)=[0,1] \wedge f_{n}\left(\frac{1}{4 n+2}\right)=f_{n}\left(\frac{1}{4 n+1}\right)=0 .
$$

Put

$$
f(x)=\left\{\begin{array}{cll}
f_{n}(x) & \text { for } & x \in I_{n}, \quad n=1,2, \ldots \\
0 & \text { for } & x \in \mathcal{R} \backslash \bigcup_{n} I_{n}
\end{array}\right.
$$

and observe that the function $f$ is $\mathcal{T}_{i}$-continuous at 0 for $i=1$, 2 . Since

$$
\mathcal{T}=\mathcal{T}_{1} \cap \mathcal{T}_{2}=\{\mathcal{R}, \emptyset\},
$$

the function $f$ is not even $\mathcal{T}$-cliquish at 0 .

Theorem 1. Let $x \in X$ be a point. Suppose that the topologies $\mathcal{T}_{1}$ and $\mathcal{T}_{2}$ satisfy to the following condition

$$
\forall_{x \in A \in \mathcal{T}_{1}} \forall_{x \in B \in \mathcal{T}_{2}} \exists_{x \in E \in \mathcal{T}} E \subset A \cup B .
$$

If a function $f: X \mapsto \mathcal{R}$ is $\mathcal{T}_{i}$-continuous at the point $x$ for $i=1,2$ then $f$ is also $\mathcal{T}$-continuous at $x$.

Proof. Fix a positive real $\eta$. From $\mathcal{T}_{i}$-continuity of the function $f$ at $x$, $i=1,2$, follows that there are sets $A_{i} \in \mathcal{T}_{i}$ such that $x \in A_{i}$ and for each point $t \in A_{i}$ the inequality

$$
|f(t)-f(x)|<\eta
$$


holds. By the condition (1) there is a set $E \in \mathcal{T}$ with

$$
x \in E \subset A_{1} \cup A_{2} .
$$

Evidently,

$$
|f(t)-f(x)|<\eta
$$

for all points $t \in E$ and the proof is completed.

We will show the necessity of condition (1) for Theorem 1 to hold.

Remark 1. Let $x \in X$ be a point. If there are sets $A_{i} \in \mathcal{T}_{i}, i=1,2$, such that $x \in A_{1} \cap A_{2}$ and

$$
\forall_{x \in A \in \mathcal{T}} A \backslash\left(A_{1} \cup A_{2}\right) \neq \emptyset,
$$

then there is a function $f: X \mapsto \mathcal{R}$ which is $\mathcal{T}_{i}$-continuous at $x$ for $i=1,2$ and which is not $\mathcal{T}$-continuous at $x$.

Proof. Put

$$
f(x)=\left\{\begin{array}{lll}
0 & \text { for } & x \in A_{1} \cup A_{2} \\
1 & \text { for } \quad x \in X \backslash\left(A_{1} \cup A_{2}\right)
\end{array}\right.
$$

and observe that $f$ is $\mathcal{T}_{i}$-continuous at $x$ for $i=1,2$. Since

$$
\forall \emptyset \neq A \in \mathcal{T} \operatorname{Osc}_{A \cup\{x\}} f=1,
$$

the function $f$ is not even $\mathcal{T}$-quasicontinuous at $x$. This completes the proof.

\section{Quasicontinuity}

Example 2. Let $X=\mathcal{R}$ and let the sets $U, V$ be the same as these from Example 1. Denote by $\mathcal{T}_{1}$ and $\mathcal{T}_{2}$ the topologies generated by family

$$
\{U \cap(0, r) ; r>0\}
$$

and respectively by the family

$$
\{V \cap(0, r) ; r>0\} .
$$

Let the function $f$ be the same as that from Example 1. Then the topologies $\mathcal{T}_{1}$ and $\mathcal{T}_{2}$ satisfy to the condition (1) from Theorem 1 , the function $f$ is $\mathcal{T}_{i}$-quasicontinuous at the point 0 (even everywhere) for $i=1,2$, but $f$ is not $\mathcal{T}$-quasicontinuos at 0 (it is not even $\mathcal{T}$-cliquish at 0 ). 
So, Theorem 1 is not true for quasicontinuity.

For the case of quasicontinuity we recall the following notion: A set $A \subset X$ is said $\tau$-semiopen if

$$
A \subset \operatorname{cl}_{\tau}\left(\operatorname{int}_{\tau}(A)\right),
$$

where $\mathrm{cl}_{\tau}$ and int $\tau$ respectively denote the operations of the closure and the interior with respect to the topology $\tau$.

Let $S(\tau)$ be the family of all $\tau$-semiopen sets $A \subset X$.

It is well known ([3]) that a function $f: X \mapsto \mathcal{R}$ is $\tau$-quasicontinuous at a point $x$ if and only if for every positive real $\eta$ there is a $\tau$-semiopen set $A \subset X$ containing $x$ such that for each point $t \in A$ the inequality

$$
|f(t)-f(x)|<\eta
$$

is true.

Theorem 2. Let $x \in X$ be a point. Suppose that the topologies $\mathcal{T}_{1}$ and $\mathcal{T}_{2}$ satisfy to the following condition

$$
\forall_{x \in A \in S\left(\mathcal{T}_{1}\right)} \forall_{x \in B \in S\left(\mathcal{T}_{2}\right)} \exists_{\emptyset \neq E \in \mathcal{T}} E \subset A \cup B .
$$

If a function $f: X \mapsto \mathcal{R}$ is $\mathcal{T}_{i}$-quasicontinuous at $x$ for $i=1,2$, then $f$ is also $\mathcal{T}$-quasicontinuous at $x$.

Proof. Fix a positive real $\eta$ and a set $A \in \mathcal{T}$ containing $x$. By the $\mathcal{T}_{i^{-}}$ quasicontinuity of the function $f$ at $x$ for $i=1,2$, there are nonempty sets

$$
A_{i} \in S\left(\mathcal{T}_{i}\right), \quad i=1,2,
$$

containing $x$ and such that for all points $t \in A_{i}, i=1,2$, the inequality

$$
|f(t)-f(x)|<\eta
$$

holds. Observe that

$$
A \cap A_{i} \in S\left(\mathcal{T}_{i}\right)
$$

for $i=1,2$. By the condition (2) there is a nonempty $\mathcal{T}$-open set

$$
B \subset A \cap\left(A_{1} \cup A_{2}\right) .
$$

Evidently,

$$
\forall_{t \in B}|f(t)-f(x)|<\eta .
$$

So, the proof is completed. 
Remark 2. Let $x \in X$ be a point. Suppose that there are nonempty sets $A_{i} \in S\left(\mathcal{T}_{i}\right), i=1,2$, containing $x$ and such that

$$
\forall \emptyset \neq A \in \mathcal{T} A \backslash\left(A_{1} \cup A_{2}\right) \neq \emptyset
$$

Then there is a function $f: X \mapsto \mathcal{R}$ which is $\mathcal{T}_{i}$-quasicontinuous at the point $x$ for $i=1,2$ and which is not $\mathcal{T}$-quasicontinuous at the point $x$.

ProOF. The construction of such a function is the same as the construction of the function $f$ in the proof of Remark 1 .

Observe that the case of quasicontinuity is different from the case of continuity. If a function $f$ is $\mathcal{T}$-continuous at a point $x$ then it is also $\mathcal{T}_{i}$-continuous at $x$ for $i=1,2$. For quasicontinuity the situation is different.

Example 3. Let $\mathcal{T}_{1}=\mathcal{T}_{e}^{+}$and respectively $\mathcal{T}_{2}=\mathcal{T}_{e}^{-}$be the topologies generated by the families

$$
\{[x, x+r) ; x \in \mathcal{R}, \quad r>0\}
$$

and

$$
\{(x-r, x] ; x \in \mathcal{R}, \quad r>0\}
$$

Then

$$
\mathcal{T}=\mathcal{T}_{1} \cap \mathcal{T}_{2}=\mathcal{T}_{e}
$$

and the function

$$
f(t)= \begin{cases}0 & \text { for } t \geq 0 \\ 1 & \text { for } t<0\end{cases}
$$

is $\mathcal{T}$-quasicontinuous at 0 and is not $\mathcal{T}_{2}$-quasicontinuous at 0. Analogously, the function

$$
g(t)=f(-t), \quad t \in \mathcal{R},
$$

is $\mathcal{T}$-quasicontinuous at 0 and is not $\mathcal{T}_{1}$-quasicontinuous at 0.

Theorem 3. Let $x \in X$ be a point. Then the following conditions are equivalent:

$\left(2^{\prime}\right)$ every function $f: X \mapsto \mathcal{R}$ which is $\mathcal{T}$-quasicontinuous at $x$ is also $\mathcal{T}_{i}$-quasicontinuous at $x$ for $i=1,2$;

$\left(2^{\prime \prime}\right)$ every $\mathcal{T}$-semiopen set containing $x$ contains also $\mathcal{T}_{i}$-semiopen sets $U_{i}, i=1,2$, containing $x$. 
Proof. $\left(2^{\prime}\right) \Rightarrow\left(2^{\prime \prime}\right)$. If $\left(2^{\prime \prime}\right)$ does not hold then there are a $\mathcal{T}$-semiopen set $U$ containing $x$ and an index $i \leq 2$ such that for every $\mathcal{T}_{i}$-semiopen set $V$ containing $x$ the relation

$$
V \backslash U \neq \emptyset
$$

holds. Let

$$
f(t)= \begin{cases}0 & \text { for } t \in U \\ 1 & \text { for } t \in X \backslash U\end{cases}
$$

Then $f$ is $\mathcal{T}$-quasicontinuous at $x$, but it is not $\mathcal{T}_{i}$-quasicontinuous at $x$. So, we obtain a contradiction with $\left(2^{\prime}\right)$.

$\left(2^{\prime \prime}\right) \Rightarrow\left(2^{\prime}\right)$. Suppose that a function $f: X \mapsto \mathcal{R}$ is $\mathcal{T}$-quasicontinuous at $x$. Fix a positive real $\eta$ and an index $i \leq 2$. From the $\mathcal{T}$-quasicontinuity of $f$ at $x$ follows the existence of an $\mathcal{T}$-semiopen set $U$ containing $x$ and such that

$$
|f(t)-f(x)|<\eta
$$

for each point $t \in U$. By $\left(2^{\prime \prime}\right)$ there is a $\mathcal{T}_{i}$-semiopen set $V \subset U$ containing $x$. Since

$$
|f(t)-f(x)|<\eta
$$

for all points $t \in V$, the function $f$ is $\mathcal{T}_{i}$-quasicontinuous at $x$ and the proof is completed.

\section{Cliquishness}

Remark 3. Assume all hypotheses of Remark 2. Moreover suppose that for each nonempty set $A \in \mathcal{T}$ there are sets $B, D$ which are dense (with respect to the topology $\mathcal{T}$ ) in $A$ and such that

$$
B \cap D=\emptyset \wedge B \cup D=A .
$$

Then there is a function $f: X \mapsto \mathcal{R}$ which is $\mathcal{T}_{i}$-quasicontinuous at the point $x$ for $i=1,2$ and which is not $\mathcal{T}$-cliquish at $x$.

Proof. If

$$
E=\operatorname{int} \mathcal{T}\left(X \backslash\left(A_{1} \cup A_{2}\right)\right) \neq \emptyset
$$

then let

$$
E=B \cup D,
$$

where the sets $B, D$ are disjoint and dense (with respect to the topology $\mathcal{T}$ ) in $E$. If $E=\emptyset$ we put

$$
B=X \backslash\left(A_{1} \cup A_{2}\right) .
$$


Define

$$
f(x)=\left\{\begin{array}{lll}
1 & \text { for } & x \in B \\
0 & \text { for } & x \in X \backslash B .
\end{array}\right.
$$

Then $f$ is $\mathcal{T}_{i}$-quasicontinuous at $x$ for $i=1,2$, but $f$ is not $\mathcal{T}$-cliquish at $x$.

The following example shows that all hypotheses of Remark 3 are essential.

Example 4. Let

$$
\begin{aligned}
& X=\{0,1,2,3\}, \\
& \mathcal{T}_{1}=\{\emptyset,\{0,1\},\{3\},\{0,1,3\}, X\}, \text { and } \mathcal{T}_{2}=\{\emptyset,\{0,2\},\{3\},\{0,2,3\}, X\} .
\end{aligned}
$$

Then

$$
\mathcal{T}=\{\emptyset,\{3\}, X\}
$$

Let $f: X \mapsto \mathcal{R}$ be a function which is $\mathcal{T}_{i}$-quasicontinuous at the point 0 for $i=1,2$. Then the function $f$ is also $\mathcal{T}_{i}$-continuous at 0 for $i=1,2$ and

$$
f(0)=f(1)=f(2) .
$$

If $0 \in A \in \mathcal{T}$, then

$$
A=X \wedge\{3\} \subset A
$$

and $\operatorname{osc}_{\{3\}} f=0$. So $f$ is $\mathcal{T}$-cliquish at the point 0 .

Moreover,

$$
\begin{aligned}
& 0 \in U=\{0,1\} \in \mathcal{T}_{1}, \\
& 0 \in W=\{0,2\} \in \mathcal{T}_{2}, \text { and } \forall_{\emptyset \neq A \in \mathcal{T}} A \backslash(U \cup W) \neq \emptyset .
\end{aligned}
$$

Theorem 4. Let $x \in X$ be a point. Suppose that

(3) there is a set $A \in \mathcal{T}$ containing $x$ and such that for all nonempty sets $A_{i} \in \mathcal{T}_{i}, i=1,2$, contained in $A$ there exists a nonempty set $D \in \mathcal{T}$ contained in $A_{1} \cup A_{2}$ and for all nonempty disjoint sets $B_{i} \in \mathcal{T}_{i}$, $i=1,2$, there are an index $i \leq 2$ and a nonempty set $E \in \mathcal{T}$ with

$$
E \subset B_{1} \vee E \subset B_{2}
$$

If a function $f: X \mapsto \mathcal{R}$ is $\mathcal{T}_{i}$-cliquish at the point $x$ for $i=1,2$, then $f$ is also $\mathcal{T}$-cliquish at $x$. 
Proof. Fix a positive real $\eta$ and a set $U$ with

$$
x \in U \in \mathcal{T} .
$$

Let a set $A$ satisfies to the condition (3). The $\mathcal{T}_{i}$-cliquishness of $f$ at $x$ implies the existence of a nonempty sets $B_{i} \subset U \cap A, i=1,2$, with

$$
\operatorname{osc}_{B_{i}} f<\frac{\eta}{2} \wedge B_{i} \in \mathcal{T}_{i}, \quad i=1,2 .
$$

Observe that

$$
B_{1} \cap B_{2} \neq \emptyset \Rightarrow \operatorname{osc}_{B_{1} \cup B_{2}} f<\eta .
$$

By (3) there is a nonempty set $E \in \mathcal{T}$ such that:

$$
B_{1} \cap B_{2}=\emptyset \Rightarrow \exists_{i \leq 2} E \subset B_{i}
$$

or

$$
B_{1} \cap B_{2} \neq \emptyset \Rightarrow E \subset B_{1} \cup B_{2} .
$$

Since in both cases osc $E f<\eta$, the proof is completed.

Remark 4. Let $x \in X$ be a point. Suppose that the topologies $\mathcal{T}, \mathcal{T}_{1}$ and $\mathcal{T}_{2}$ are such that for each point $t \neq x$ there are sets $U_{1} \in \mathcal{T}_{1}, U_{2} \in \mathcal{T}_{2}$ and $U_{3} \in \mathcal{T}$ for which $U_{1} \cap U_{3}=\emptyset, U_{2} \cap U_{3}=\emptyset, x \in U_{3}$ and $t \in U_{1} \cap U_{2}$. Moreover we assume that there is a countable base of neighborhoods of $x$ for the topology $\mathcal{T}$ and that for which set $A \in \mathcal{T}$ containing $x \in X$ there are nonempty sets $A_{i}(A) \in \mathcal{T}_{i}, i=1,2$, such that:

$$
\begin{gathered}
A_{1}(A) \cap A_{2}(A) \neq \emptyset \Rightarrow \forall \forall \neq B \in \mathcal{T} B \backslash\left(A_{1}(A) \cup A_{2}(A)\right) \neq \emptyset, \\
A_{1}(A) \cap A_{2}(A)=\emptyset \Rightarrow \forall_{\emptyset \neq B \in \mathcal{T}} \forall_{i \leq 2} B \backslash A_{i}(A) \neq \emptyset,
\end{gathered}
$$

and

$$
G=X \backslash \bigcup\left\{A_{1}(A) \cup A_{2}(A) ; x \in A \in \mathcal{T}\right\} \backslash\{x\}=H \cup K,
$$

where $H \cap K=\emptyset$ and $H, K$ are dense in $G$ with respect to the topology $\mathcal{T}$. Then there is a function $f: X \mapsto \mathcal{R}$ which is $\mathcal{T}_{i}$-cliquish at the point $x$ for $i=1,2$ and which is not $\mathcal{T}$-cliquish at $x$.

Proof. At the beginning we assume that $\{x\}$ is not in $\mathcal{T}_{i}$ for $i=1,2$. Let

$$
W_{1}, \ldots, W_{n}, \ldots
$$

be an enumeration of all elements of some basis of neighborhoods of $x$ in the topology $\mathcal{T}$. Let

$$
A_{i}\left(W_{1}\right) \in \mathcal{T}_{i}, \quad i=1,2,
$$


be nonempty sets satisfying our hypothesis for $A=W_{1}$. Since in this case there are points $t_{i} \in A_{i}\left(W_{1}\right), i=1,2$, we can find nonempty sets $V_{1, i} \in \mathcal{T}_{i}$, $i \leq 2$, and $V_{1,3} \in \mathcal{T}$ with $t_{i} \in V_{1, i}, i \leq 2, x \in V_{1,3}$, and

$$
V_{1,3} \cap\left(V_{1,1} \cup V_{1,2}\right)=\emptyset .
$$

If $V_{1,1} \cap V_{1,2}=\emptyset$ we put

$$
f(t)=(-1)^{i}, \quad t \in V_{1, i}, \quad i \leq 2 .
$$

In the contrary case, where $V_{1,1} \cap V_{1,2} \neq \emptyset$, we put

$$
f(t)=1, \quad t \in V_{1,1} \cup V_{1,2} .
$$

Analogously, for $n>1$ we find nonempty sets $V_{n, i} \in \mathcal{T}_{i}, i \leq 2$, and $V_{n, 3} \in \mathcal{T}$ such that:

$$
\begin{gathered}
x \in V_{n, 3} \subset X \backslash\left(V_{n, 1} \cup V_{n, 2}\right), \\
V_{n, 1} \cup V_{n, 2} \subset W_{n} \cap V_{1,3} \cap \ldots \cap V_{n-1,3}, \\
V_{n, 1} \cap V_{n, 2} \neq \emptyset \Rightarrow \forall_{\emptyset \neq B \in \mathcal{T} B} \backslash\left(V_{n, 1} \cup V_{n, 2}\right) \neq \emptyset,
\end{gathered}
$$

and

$$
V_{n, 1} \cap V_{n, 2}=\emptyset \Rightarrow \forall \emptyset \neq B \in \mathcal{T} \forall_{i \leq 2} B \backslash V_{n, i} \neq \emptyset .
$$

If $V_{n, 1} \cap V_{n, 2}=\emptyset$ then we put

$$
f(t)=(-1)^{i} n, \quad t \in V_{n, i}, \quad i \leq 2 .
$$

In the contrary case, where $V_{n, 1} \cap V_{n, 2} \neq \emptyset$ we put

$$
f(t)=n, \quad t \in V_{n, 1} \cup V_{n, 2} .
$$

Moreover, let

$$
\begin{aligned}
& f(x)=0, \\
& f(t)=0, \quad t \in H, \text { and } f(t)=\frac{1}{2}
\end{aligned}
$$

at all other points of the space $X$.

Observe that the function $f$ satisfies all requirements.

Evidently,

$$
\neg\left(\{x\} \in \mathcal{T}_{1} \cap \mathcal{T}_{2}\right) .
$$

In the case, where $\{x\} \in \mathcal{T}_{i}$ for $i=1$ or $i=2$, the construction of such a function $f$ is simpler, since starting from some index $n$ we find only one set $V_{k, i_{0}}$ instead a pair $\left(V_{k, 1}, V_{k, 2}\right)$. 
Theorem 5. Let $x \in X$ be a point. Suppose that

(a) all sets $A$ with

$$
x \in A \in \mathcal{T}_{1} \cup \mathcal{T}_{2}
$$

contains some sets $B \in \mathcal{T}$ with $x \in B$.

Then every function $f: X \mapsto \mathcal{R}$ which is $\mathcal{T}$-cliquish at $x$ is also $\mathcal{T}_{i}$-cliquish at $x$ for $i=1,2$.

Proof. Fix an index $i \leq 2$, a positive real $\eta$ and a set $U \in \mathcal{T}_{i}$ with $x \in U$. By our hypothesis there is a set $B \in \mathcal{T}$ such that

$$
x \in B \subset U .
$$

Since $f$ is $\mathcal{T}$-cliquish at $x$, there is a nonempty set $V \in \mathcal{T}$ such that

$$
V \subset B \wedge \operatorname{osc}_{V} f<\eta \text {. }
$$

So,

$$
V \subset U \wedge V \in \mathcal{T}_{i} \wedge \operatorname{osc}_{V} f<\eta,
$$

and the proof is completed.

The next example shows that hypothesis (a) from the last theorem is not necessary.

Example 5. Let

$$
\begin{aligned}
& X=\{0,1,2,3\}, \\
& \mathcal{T}_{1}=\{\emptyset, X,\{1\}\}, \text { and } \mathcal{T}_{2}=\{\emptyset, X,\{2\}\} .
\end{aligned}
$$

Then

$$
\mathcal{T}=\{\emptyset, X\},
$$

every function $f: X \mapsto \mathcal{R}$ which is $\mathcal{T}$-cliquish at $x$ is constant and the condition (a) is not satisfied.

The proof of the next theorem gives a characterization of the cliquishness.

Remark 5. Let $\tau$ be a topology of subsets of $X$ and let $x \in X$ be a point. A function $f: X \mapsto \mathcal{R}$ is $\tau$-cliquish at $x$ if and only if for each positive real $\eta$ there are $\tau$-open sets $A_{s}, s \in S$, where $S$ is a set of indexes, such that $x$ belongs to the $\tau$-closure $\mathrm{cl}_{\tau}\left(\bigcup_{s \in S} A_{s}\right)$ of the set $\bigcup_{s \in S} A_{s}$ and for every $s \in S$ the inequality $\operatorname{osc}_{A_{s}} f<\eta$ is true. 
Proof. Fix a positive real $\eta$. If $f$ is $\tau$-cliquish at $x$ then for every set $U \in \tau$ containing $x$ there is a nonempty $\tau$-open set $B(U) \subset U$ with osc $B(U) f<\eta$. If

$$
\left\{U_{s} ; s \in S\right\}
$$

is a directed family of all $\tau$-open sets contained the point $x$, then the family

$$
\left\{A_{s}=B\left(U_{s}\right) ; s \in S\right\}
$$

satisfies all the required conditions.

The proof of the second implication is evident.

Theorem 6. Let $x \in X$ be a point. Suppose that the topologies $\mathcal{T}_{1}$ and $\mathcal{T}_{2}$ satisfy the following condition

$\left(3^{\prime}\right) \quad$ for each $\mathcal{T}$-open set $A$ such that $x \in \operatorname{cl}_{\mathcal{T}}(A)$ and for each index $i \leq 2$ the point $x \in \operatorname{cl}_{\mathcal{T}_{i}}(A)$.

If a function $f: X \mapsto \mathcal{R}$ is $\mathcal{T}$-cliquish at $x$ then it is also $\mathcal{T}_{i}$-cliquish at $x$ for $i=1,2$.

Proof. Fix a positive integer $i \leq 2$ and a positive real $\eta$. Since $f$ is $\mathcal{T}$-cliquish at $x$, by the last Remark there are nonempty sets $A_{s} \in \mathcal{T}$, where $S$ is a set of indexes, such that

$$
x \in \operatorname{cl} \mathcal{T}\left(\bigcup_{s \in S} A_{s}\right)
$$

and for every $s \in S$ the inequality osc $A_{s} f<\eta$ is true.

From $\left(3^{\prime}\right)$ follows the existence of a $\mathcal{T}_{i}$-open set $D \subset \bigcup_{s \in S} A_{s}$ with

$$
x \in \operatorname{cl}_{\mathcal{T}_{i}}(D) .
$$

For $s \in S$ the sets

$$
D_{s}=D \cap A_{s} \in \mathcal{T}_{i} .
$$

Let

$$
S^{\prime}=\left\{s \in S ; D_{s} \neq \emptyset\right\} .
$$

Since for $s \in S^{\prime}$ the inequality

$$
\operatorname{osc}_{D_{s}} f<\eta
$$

is true and since

$$
x \in \operatorname{cl}_{\mathcal{T}_{i}}\left(\bigcup_{s \in S^{\prime}} D_{s}\right),
$$

by the last Remark $f$ is $\mathcal{T}_{i}$-cliquish at $x$. So, the proof is completed. 
Remark 6. Let $x \in X$ be a point. Suppose that there are an index $i \leq 2$, a set $V \in \mathcal{T}_{i}$, disjoint sets $Y, Z \subset V$ and a set $U \in \mathcal{T}$ such that

$$
U \cap V=\emptyset \wedge x \in \operatorname{cl}_{\mathcal{T}}(U) \cap V,
$$

and

$$
x \in Y \wedge \operatorname{cl}_{\mathcal{T}_{i}}(Y)=\operatorname{cl}_{\mathcal{T}_{i}}(Z)=\operatorname{cl}_{\mathcal{T}_{i}}(V) .
$$

Then there is a function $f: X \mapsto \mathcal{R}$ which is $\mathcal{T}$-cliquish at $x$ and which is not $\mathcal{T}_{i}$-cliquish at $x$.

Proof. The function

$$
f(t)=\left\{\begin{array}{lll}
1 & \text { if } & t \in Z \\
0 & \text { if } & t \in X \backslash Z
\end{array}\right.
$$

is $\mathcal{T}$-cliquish at $x$, but it is not $\mathcal{T}_{i}$-cliquish at $x$. So, the proof is completed.

The following example shows the importance of all hypothesis of the last remark.

Example 6. Let

$$
\begin{aligned}
& X=\{0,1,2\}, \\
& \mathcal{T}_{1}=\{\emptyset, X,\{1\}\}, \text { and } \mathcal{T}_{2} \quad=2^{X} .
\end{aligned}
$$

Then $\mathcal{T}=\mathcal{T}_{1}$ and for

$$
x=0 \wedge A=\{1\} \wedge B=\{0\}
$$

we have

$$
A \in \mathcal{T} \wedge B \in \mathcal{T}_{2} \wedge A \cap B=\emptyset
$$

and

$$
x \in B \wedge x \in \operatorname{cl}_{\mathcal{T}}(A) .
$$

Moreover, each function $f: X \mapsto \mathcal{R}$ is $\mathcal{T}_{2}$-continuous at the point 0 , because $\{0\} \in \mathcal{T}_{2}$. So, every function $f: X \mapsto \mathcal{R}$ which is $\mathcal{T}$-cliquish at 0 is also $\mathcal{T}_{2}$-cliquish at 0 .

\section{References}

[1] R. Engelking, General Topology, Warsaw PWN, 1976.

[2] J. L. Kelly, General Topology, New York 1955.

[3] T. Neubrunn, Quasi-continuity, Real Anal. Exch. 14 (1988-89), 259-306. 